\title{
Dynamics of the Free Surface of a Conducting Liquid in a Near-Critical Electric Field
}

\author{
Nikolay M. Zubarev* and Olga V. Zubareva \\ Institute of Electrophysics, Ural Branch, Russian Academy of Sciences, \\ 106 Amundsen Street, 620016 Ekaterinburg, Russia
}

\begin{abstract}
Near-critical behavior of the free surface of an ideally conducting liquid in an external electric field is considered. Based on an analysis of three-wave processes using the method of integral estimations, sufficient criteria for hard instability of a planar surface are formulated. It is shown that the higher-order nonlinearities do not saturate the instability, for which reason the growth of disturbances has an explosive character.
\end{abstract}

\section{INTRODUCTION}

The electrohydrodynamic instability of the free surface of a conducting liquid in a strong electric field [1, 2] is responsible for many physical processes, such as the initiation and maintenance of emission of charged particles, vacuum breakdown, vacuum discharge, etc. The interaction of an electric field and charges induced by this field on the surface of a liquid (liquid metal) leads to a growth of surface disturbances and the formation of regions with a significant curvature [3, 4, 5]. The dispersion law for the waves on a planar surface of an ideally conducting liquid in an external electric field of strength $E$ has the following form 6]:

$$
\omega^{2}=g|\mathbf{k}|+\frac{\alpha}{\rho}|\mathbf{k}|^{3}-\frac{E^{2}}{4 \pi \rho}|\mathbf{k}|^{2}
$$

where $\omega$ is the frequency, $\mathbf{k}$ is the wave vector, $g$ is the acceleration of gravity, $\alpha$ is the surface tension coefficient, and $\rho$ is the density of the medium.

It is seen from Eq. (1) that if the condition

$$
E^{2}<E_{c}^{2}=8 \pi \sqrt{g \alpha \rho}
$$

*Electronic address: nick@ami.uran.ru 
is fulfilled, then $\omega^{2}>0$ at any $|\mathbf{k}|$ and, consequently, the surface disturbances do not grow with time. If the magnitude of the field $E$, which plays the role of an external governing parameter, exceeds a certain critical value $E_{c}$, then there arises a region of wave vectors $|\mathbf{k}|$, for which $\omega^{2}<0$, which corresponds to an aperiodic instability. Thus, the condition $E>E_{c}$ is a criterion for the surface instability with respect to infinitely small disturbances of the surface shape and of the field of velocities.

It was shown in 7, , 8, 9], where liquids with various physical properties have been considered, that a nonlinear interaction of three standing waves that form a hexagonal structure can lead to a hard excitation of the instability of a charged surface. In our case, this means that, even at subcritical fields $\left(E<E_{c}\right)$ a disturbance of a sufficient magnitude can break the equilibrium of a planar surface. In this connection, there arises a need of constructing criteria for the instability of a charged surface of a conducting liquid with respect to perturbations of a finite magnitude, i.e., criteria that will permit one, proceeding from some initial data such as the shape of the surface and the distribution of velocities, to answer the question of whether or not the initial perturbation will lead to the loss of the stability of a planar boundary and, as a consequence, to an explosive growth of cusplike structures. This work is devoted to constructing such criteria using the method of integral estimations that was applied previously to obtain the conditions of collapse for the nonlinear Schrodinger equation [10, 11], nonlinear Klein-Gordon equation [12, 13], and various modifications of the Boussinesq equation [14].

In Section 2, we give equations of the vertex-free motion of an ideally conducting liquid with a free surface in an electric field and give their Hamiltonian formulation. In Section 3, the theory of perturbations in a small parameter, namely, in the characteristic angle of the surface slope, is constructed up to fourth-order terms in the Hamiltonian. The analysis of the surface dynamics is significantly simplified in the case of small "supercriticalities" (i.e., if the field only slightly exceeds the critical value $E_{c}$ )

$$
\varepsilon=\left(E^{2}-E_{c}^{2}\right) / E_{c}^{2}, \quad|\varepsilon| \ll 1,
$$

when only perturbations with wave numbers close to $k_{0}=\sqrt{g \rho / \alpha}$ increase (this value of the wave number corresponds to the so-called dominant harmonic of surface perturbations). This permits us in Section 4 to construct a set of amplitude equations for describing the nonlinear interaction of three standing waves that form a hexagonal structure which is the 
main interaction at near-critical values of the field $E$. In Section 5, we extend the method of integral estimations for several interacting nonlinear waves. By using this method, one can pass from a set of partial differential equations for complex amplitudes $A_{1}, A_{2}$ and $A_{3}$ to to a second-order differential inequality for the norm

$$
X=\int\left(\left|A_{1}\right|^{2}+\left|A_{2}\right|^{2}+\left|A_{3}\right|^{2}\right) d^{2} r,
$$

by analyzing which we obtain a number of sufficient criteria of hard excitation of an electrohydrodynamic instability of a charged surface. Note that most of them refer to subcritical values of the external electric field, when the surface is stable in a linear approximation, while the development of the instability is related to three-wave processes. In Section 6, we show, on the example of one-dimensional and square lattices of surface perturbations for which the three-wave interactions degenerate, that the higher-order wave processes do not saturate the instability but, on the contrary, lead to an explosive growth of amplitudes.

\section{STARTING EQUATIONS}

Consider the potential motion of an ideal conducting liquid of infinite depth placed in an external uniform electric field of strength $E$. Assume that the fieldstrength vector is directed along the $z$ axis and, correspondingly, in the unperturbed state the boundary of the liquid is a planar horizontal surface $z=0$. Let the function $\eta(x, y, t)$ specify the deviation of the boundary from the flatness, i.e., the region occupied by the liquid is restricted by a free surface $z=\eta$.

The velocity potential for an incompressible liquid $\Phi$ satisfies Laplace's equation

$$
\nabla^{2} \Phi=0
$$

with the following conditions at the metal-vacuum boundary and at infinity:

$$
\begin{gathered}
\frac{\partial \Phi}{\partial t}+\frac{(\nabla \Phi)^{2}}{2}=\frac{(\nabla \varphi)^{2}-E^{2}}{8 \pi \rho}+\frac{\alpha}{\rho} \nabla_{\perp} \cdot \frac{\nabla_{\perp} \eta}{\sqrt{1+\left(\nabla_{\perp} \eta\right)^{2}}}-g \eta, \quad z=\eta, \\
\Phi \rightarrow 0, \quad z \rightarrow-\infty,
\end{gathered}
$$

where $\varphi$ is the electric-field potential. 
The first term on the right-hand side of the dynamic boundary condition (nonstationary Bernoulli equation) is responsible for the electrostatic pressure, the second term determines the capillary pressure, and the third term takes into account the effect of the gravitational field. The time evolution of the free surface is determined by the kinematic relationship (condition of non- flowing of the liquid through its boundary)

$$
\frac{\partial \eta}{\partial t}=\frac{\partial \Phi}{\partial z}-\nabla_{\perp} \eta \cdot \nabla_{\perp} \Phi, \quad z=\eta
$$

Finally, the electric-field potential $\varphi$ in the absence of spatial charges satisfies Laplace's equation

$$
\nabla^{2} \varphi=0
$$

which should be solved simultaneously with the condition of the equipotentiality of the boundary of the conducting liquid and the condition of the uniformity of the field at an infinite distance from the surface:

$$
\begin{gathered}
\varphi=0, \quad z=\eta, \\
\varphi \rightarrow-E z, \quad z \rightarrow \infty .
\end{gathered}
$$

Note that the above-written equations of motion have a Hamiltonian structure and the functions $\eta(x, y, t)$ and $\psi(x, y, t)=\left.\Phi\right|_{z=\eta}$ are canonically conjugated quantities [15]

$$
\frac{\partial \psi}{\partial t}=-\frac{\delta H}{\delta \eta}, \quad \frac{\partial \eta}{\partial t}=\frac{\delta H}{\delta \psi},
$$

where the Hamiltonian $H$ coincides to an accuracy of a constant with the total energy of the system

$$
\begin{gathered}
H=\int_{z \leq \eta} \frac{(\nabla \Phi)^{2}}{2} d^{3} r-\int_{z \geq \eta} \frac{(\nabla \varphi)^{2}}{8 \pi \rho} d^{3} r \\
+\int\left[\frac{g \eta^{2}}{2}+\frac{\alpha}{\rho}\left(\sqrt{1+\left(\nabla_{\perp} \eta\right)^{2}}-1\right)\right] d^{2} r .
\end{gathered}
$$

For a further consideration of the problem, it is convenient to represent the Hamiltonian in the form of a surface integral. We introduce a perturbation of the electric-field potential $\tilde{\varphi}=\varphi+E z$. It can easily be shown that the perturbed potential $\tilde{\varphi}$ satisfies Laplace's equation

$$
\nabla^{2} \tilde{\varphi}=0
$$


with conditions

$$
\begin{aligned}
& \tilde{\varphi}=E \eta, \quad z=\eta, \\
& \tilde{\varphi} \rightarrow 0, \quad z \rightarrow \infty,
\end{aligned}
$$

from which it is seen that the perturbation introduced by the surface $z=\eta$ into the distribution of the electric field decays as $z \rightarrow \infty$. Taking into account that, in view of the incompressibility of the liquid, a relation $\left.\int \tilde{\varphi}\right|_{z=\eta} d^{2} r=0$ is valid, and neglecting terms whose variation does not contribute to the equation of motion, we obtain, using the first Green's formula,

$$
H=\int_{s}\left[\frac{\psi}{2} \frac{\partial \Phi}{\partial n}+\frac{E \eta}{8 \pi \rho} \frac{\partial \tilde{\varphi}}{\partial n}\right] d s+\int\left[\frac{g \eta^{2}}{2}+\frac{\alpha}{\rho}\left(\sqrt{1+\left(\nabla_{\perp} \eta\right)^{2}}-1\right)\right] d^{2} r,
$$

where $d s$ is the surface differential and $\partial / \partial n$ denotes the derivative in the direction of the normal to the surface $z=\eta$.

By eliminating the normal derivatives of the potentials $\tilde{\varphi}$ and $\Phi$, we can reduce the expression for the Hamiltonian to the form

$$
\begin{gathered}
H=\int\left[\left.\frac{\psi}{2}\left(\Phi_{z}-\nabla \eta \cdot \nabla_{\perp} \Phi\right)\right|_{z=\eta}+\left.\frac{E \eta}{8 \pi \rho}\left(\tilde{\varphi}_{z}-\nabla \eta \cdot \nabla_{\perp} \tilde{\varphi}\right)\right|_{z=\eta}\right] d^{2} r \\
+\int\left[\frac{g \eta^{2}}{2}+\frac{\alpha}{\rho}\left(\sqrt{1+\left(\nabla_{\perp} \eta\right)^{2}}-1\right)\right] d^{2} r
\end{gathered}
$$

which is more suitable for further transformations.

\section{SMALL-ANGLE APPROXIMATION}

Our further problem is to eliminate the spatial variable $z$ from the equations of motion, i.e., to pass from the initial three-dimensional equations to two-dimensional ones. To do this, we should write the integrand in Eq. (8) through the canonical variables $\eta$ and $\psi$. Then, there arises a need to solve Eq. (5) with conditions (6) and (7) as well as Eq. (2) with the condition

$$
\Phi=\psi, \quad z=\eta,
$$


and condition (3). We use the known solutions to Laplace's equation for the half-spaces $z<0$ and $z>0$ for functions that decay at infinity

$$
\begin{array}{cl}
\tilde{\varphi}(x, y, z) & =\frac{1}{2 \pi} \int_{-\infty}^{+\infty} \int_{-\infty}^{+\infty} \frac{z \tilde{\varphi}\left(x^{\prime}, y^{\prime}, 0\right)}{\left[\left(x^{\prime}-x\right)^{2}+\left(y^{\prime}-y\right)^{2}+z^{2}\right]^{3 / 2}} d x^{\prime} d y^{\prime}, \quad z>0, \\
\Phi(x, y, z) & =-\frac{1}{2 \pi} \int_{-\infty}^{+\infty} \int_{-\infty}^{+\infty} \frac{z \Phi\left(x^{\prime}, y^{\prime}, 0\right)}{\left[\left(x^{\prime}-x\right)^{2}+\left(y^{\prime}-y\right)^{2}+z^{2}\right]^{3 / 2}} d x^{\prime} d y^{\prime}, \quad z<0 .
\end{array}
$$

Now, we should express the magnitudes of the potentials $\tilde{\varphi}$ and $\Phi$ that enter into these relationships at the plane $z=0$ through their magnitudes at the boundary $z=\eta$, i.e., through the functions $E \eta$ and $\psi$. Let the characteristic angles of the surface slope be small: $\left|\nabla_{\perp} \eta\right| \ll 1$. In this case the potentials near the $z=0$ plane can be expanded into a power series in surface perturbation $\eta$ :

$$
\tilde{\varphi}(x, y, \eta(x, y))=\left.\sum_{n=0}^{\infty} \frac{\eta^{n}}{n !} \frac{\partial^{n} \tilde{\varphi}}{\partial z^{n}}\right|_{z=0}, \quad \Phi(x, y, \eta(x, y))=\left.\sum_{n=0}^{\infty} \frac{\eta^{n}}{n !} \frac{\partial^{n} \Phi}{\partial z^{n}}\right|_{z=0} .
$$

By differentiating Eqs. (9) and (10) with respect to $z$, we find that

$$
\left.\tilde{\varphi}_{z}\right|_{z=0}=-\left.\hat{k} \tilde{\varphi}\right|_{z=0},\left.\quad \Phi_{z}\right|_{z=0}=\left.\hat{k} \Phi\right|_{z=0},
$$

where $\hat{k}$ is the two-dimensional integral operator given by the expression

$$
\hat{k} f=-\frac{1}{2 \pi} \int_{-\infty}^{+\infty} \int_{-\infty}^{+\infty} \frac{f\left(x^{\prime}, y^{\prime}\right)}{\left[\left(x^{\prime}-x\right)^{2}+\left(y^{\prime}-y\right)^{2}\right]^{3 / 2}} d x^{\prime} d y^{\prime} .
$$

This relationship can be considered as a consequence of the fact that the Laplacian operator can formally be represented as

$$
\nabla^{2}=\left(\partial_{z}+\hat{k}\right)\left(\partial_{z}-\hat{k}\right)
$$

where the left-hand bracket corresponds to solutions that are asymptotically decay as $z \rightarrow$ $+\infty$ and the right-hand bracket corresponds to solutions that decay as $z \rightarrow-\infty$.

By eliminating the derivative with respect to $z$ from the expansions (11), we find

$$
\tilde{\varphi}(x, y, \eta(x, y))=\hat{T}_{+} \tilde{\varphi}(x, y, 0), \quad \Phi(x, y, \eta(x, y))=\hat{T}_{-} \Phi(x, y, 0)
$$

where we introduced nonlinear shear operators

$$
\hat{T}_{+}=\sum_{n=0}^{\infty} \frac{\eta^{n} \hat{k}^{n}}{n !}, \quad \hat{T}_{-}=\sum_{n=0}^{\infty} \frac{(-\eta)^{n} \hat{k}^{n}}{n !} .
$$


Let $T_{ \pm}^{-1}$ be the operators that are inverse with respect to the shear operators. Their form can be determined using the method of successive approximations

$$
T_{ \pm}^{-1}=1 \mp \eta \hat{k}-\eta^{2} \hat{k}^{2} / 2+\eta \hat{k} \eta \hat{k}+\ldots
$$

Then, we have

$$
\tilde{\varphi}(x, y, 0)=E \hat{T}_{+}^{-1} \eta(x, y), \quad \Phi(x, y, 0)=\hat{T}_{-}^{-1} \psi(x, y) .
$$

These relationships, along with Eqs. (9) and (10), specify the solutions to Laplace's equations with necessary boundary conditions in the form of infinite series. Using these solutions, we can write the various possible derivatives of the potentials $\tilde{\varphi}$ and $\Phi$ that enter into the Hamiltonian through the functions $\eta$ and $\psi$. As a result, we find

$$
\begin{aligned}
& H=\int \frac{\psi}{2}\left(\hat{T}_{+} \hat{k} \hat{T}_{+}^{-1} \psi-\nabla_{\perp} \eta \cdot \hat{T}_{+} \nabla_{\perp} \hat{T}_{+}^{-1} \psi\right) d^{2} r \\
& -\int \frac{E^{2} \eta}{8 \pi \rho}\left(\hat{T}_{-} \hat{k} \hat{T}_{-}^{-1} \eta+\nabla_{\perp} \eta \cdot \hat{T}_{-} \nabla_{\perp} \hat{T}_{-}^{-1} \eta\right) d^{2} r \\
& \quad+\int\left[\frac{g \eta^{2}}{2}+\frac{\alpha}{\rho}\left(\sqrt{1+\left(\nabla_{\perp} \eta\right)^{2}}-1\right)\right] d^{2} r .
\end{aligned}
$$

To describe the initial stages of instability development on the surface of a conducting liquid, it is sufficient to restrict ourselves by allowance for a finite number of terms in the expansion of the integrands of the functional $H$ in canonical variables. By omitting terms of higher than the fourth order of smallness for the surface perturbation $\eta$ and higher than the second order for the potential $\psi$, (this proves to be sufficient at small supercriticalities) and successively integrating by parts, we finally obtain

$$
\begin{gathered}
H=H^{(2)}+H^{(3)}+H^{(4)}, \\
H^{(2)}=\int\left[\frac{\psi \hat{k} \psi}{2}-\frac{E^{2} \eta \hat{k} \eta}{8 \pi \rho}+\frac{g \eta^{2}}{2}+\frac{\alpha(\nabla \eta)^{2}}{2 \rho}\right] d^{2} r, \\
H^{(3)}=\frac{E^{2}}{8 \pi \rho} \int \eta\left[(\nabla \eta)^{2}-(\hat{k} \eta)^{2}\right] d^{2} r, \\
H^{(4)}=-\frac{E^{2}}{8 \pi \rho} \int\left[\eta \hat{k} \eta \hat{k} \eta \hat{k} \eta+\eta \hat{k} \eta^{2} \nabla^{2} \eta\right] d^{2} r-\int \frac{\alpha(\nabla \eta)^{4}}{8 \rho} d^{2} r .
\end{gathered}
$$

These expressions, in combination with Eq. (4), represent a two-dimensional reduction of the equations of motion of a conducting liquid in an external electric field that is applicable if the condition of the smallness of the characteristic angles of the surface slope is fulfilled. 


\section{AMPLITUDE EQUATIONS}

Now, let us consider the nonlinear dynamics of the perturbations of the free surface of a conducting liquid for the case where the magnitude of the external electric field $E$ is close to its threshold value $E_{c}$, i.e., $|\varepsilon| \ll 1$. It follows from the dispersion relation (1) that, at small supercriticalities, only surface waves with wave numbers close to $k_{0}$ can be excited. The main nonlinear interaction in this case will be the three-wave interaction between the waves whose wave vectors are turned with respect to one another by an angle of $2 \pi / 3$. This can easily be understood from the conditions

$$
\mathbf{k}_{1}+\mathbf{k}_{2}+\mathbf{k}_{3}=0, \quad\left|\mathbf{k}_{1}\right|=\left|\mathbf{k}_{2}\right|=\left|\mathbf{k}_{3}\right|=k_{0} .
$$

Near the threshold, it is natural to pass to envelopes using the following substitutions:

$$
\begin{aligned}
& \eta(\mathbf{r}, t)=\sum_{j=1}^{3} A_{j}\left(x_{j}, y_{j}, t\right) e^{i \mathbf{k}_{j} \mathbf{r}}+\text { c.c. } \\
& \psi(\mathbf{r}, t)=\sum_{j=1}^{3} B_{j}\left(x_{j}, y_{j}, t\right) e^{i \mathbf{k}_{j} \mathbf{r}}+\text { c.c. }
\end{aligned}
$$

where $\mathbf{k}_{1}=\left\{k_{0}, 0\right\}, \mathbf{k}_{2}=\left\{-k_{0} / 2, \sqrt{3} k_{0} / 2\right\}, \mathbf{k}_{3}=\left\{-k_{0} / 2,-\sqrt{3} k_{0} / 2\right\}$, and $A_{j}$ and $B_{j}$ $(j=1,2,3)$ are slow functions of the variables $x_{j}$ and $y_{j}$ that form orthogonal coordinate systems with abscissa axes directed along the wave vectors $\mathbf{k}_{j}$. Such a representation for the functions $\eta$ and $\psi$ corresponds to a hexagonal structure of the perturbed surface.

Using these relationships for the perturbations $\eta$ and $\psi$ we can approximate the integral operator $\hat{k}$ that enters into the Hamiltonian (12) by a differential operator. Let us use the following property:

$$
\hat{k} e^{i \mathbf{k r}}=|\mathbf{k}| e^{i \mathbf{k r}}
$$

which is related to the fact that the Fourier transform of the operator $\hat{k}$ is equal to the modulus of the wave vector. Consider a plane wave of the form

$$
e^{i \mathbf{k r}}=e^{i\left(k_{0} x+q_{x} x+q_{y} y\right)},
$$

whose wave vector is close to $k_{0}$ (i.e., $\left|q_{x}\right| \ll k_{0}$ and $\left|q_{y}\right| \ll k_{0}$ ). The quantity $|\mathbf{k}|$ can be expanded in a series in $q_{x}$ and $q_{y}$ :

$$
|\mathbf{k}|=\sqrt{\left(k_{0}+q_{x}\right)^{2}+q_{y}^{2}} \approx k_{0}+q_{x}+\left(2 k_{0}\right)^{-1} q_{y}{ }^{2}-\left(2 k_{0}^{2}\right)^{-1} q_{x} q_{y}{ }^{2}-\left(2 k_{0}\right)^{-3} q_{y}{ }^{4} .
$$


This means that if we deal with a narrow (in the $\mathbf{k}$ space) wave packet with a carrying wave vector $\mathbf{k}=\left\{k_{0}, 0\right\}$ (which can be represented in the form $A(x, y) e^{i k_{0} x}$ ), then the operator $\hat{k}$ can be approximated as follows:

$$
\hat{k} A(x, y, t) e^{i k_{0} x} \approx\left(k_{0} A-i A_{x}-\left(2 k_{0}\right)^{-1} A_{y y}-i\left(2 k_{0}^{2}\right)^{-1} A_{x y y}-\left(2 k_{0}\right)^{-3} A_{y y y y}\right) e^{i k_{0} x}
$$

(similar relations are obtained for the amplitudes $A_{j}$ in the coordinates of $x_{j}$ and $y_{j}$ ). Then, inserting the expressions for $\eta$ and $\psi$ into the Hamiltonian (12) and performing necessary averaging, we find (to an accuracy of terms of a higher order of smallness)

$$
\begin{gathered}
H=\sum_{j=1}^{3} \int\left(k_{0}\left|B_{j}\right|^{2}-2 g \varepsilon\left|A_{j}\right|^{2}+\frac{g}{k_{0}^{2}}\left|\frac{\partial A_{j}}{\partial x_{j}}-\frac{i}{2 k_{0}} \frac{\partial^{2} A_{j}}{\partial y_{j}^{2}}\right|^{2}\right) d^{2} r \\
-3 g k_{0} \int\left(A_{1} A_{2} A_{3}+A_{1}^{*} A_{2}^{*} A_{3}^{*}\right) d^{2} r .
\end{gathered}
$$

The dynamic equations that describe the time evolution of the amplitudes $A_{j}$ and $B_{j}$ are found from the relations 16 ]

$$
A_{j_{t}}=\frac{\delta H}{\delta B_{j}^{*}}, \quad B_{j_{t}}=-\frac{\delta H}{\delta A_{j}^{*}},
$$

where $j=1,2,3$.

By varying the expression for the averaged Hamiltonian, we obtain the following equations for the amplitudes:

$$
\begin{gathered}
A_{j_{t}}=k_{0} B_{j}, \\
B_{j_{t}}=2 g k_{0} \varepsilon A_{j}+\frac{g}{k_{0}}\left(\frac{\partial}{\partial x}-\frac{i}{2 k_{0}} \frac{\partial^{2}}{\partial y^{2}}\right)^{2} A_{j}+3 g k_{0}{ }^{2} \frac{A_{1}^{*} A_{2}^{*} A_{3}^{*}}{A_{j}^{*}} .
\end{gathered}
$$

By eliminating the amplitudes $B_{j}$ from these equations and passing to dimensionless quantities using the substitutions

$$
\mathbf{r} \rightarrow \mathbf{r} /\left(\sqrt{2} k_{0}\right), \quad A_{j} \rightarrow A_{j} / k_{0}, \quad t \rightarrow t / \sqrt{2 g k_{0}}, \quad H \rightarrow 2 H g / k_{0}^{2},
$$

we obtain the following set of equations:

$$
\begin{aligned}
& A_{1 t t}=\varepsilon A_{1}+\hat{L}_{1}^{2} A_{1}+3 A_{2}^{*} A_{3}^{*} / 2, \\
& A_{2 t t}=\varepsilon A_{2}+\hat{L}_{2}^{2} A_{2}+3 A_{3}^{*} A_{1}^{*} / 2,
\end{aligned}
$$




$$
A_{3 t t}=\varepsilon A_{3}+\hat{L}_{3}^{2} A_{3}+3 A_{1}^{*} A_{2}^{*} / 2
$$

where we introduced operators

$$
\hat{L}_{j}=\frac{\partial}{\partial x_{j}}-\frac{i}{\sqrt{2}} \frac{\partial^{2}}{\partial y_{j}^{2}}, \quad j=1,2,3 .
$$

The Hamiltonian corresponding to these amplitude equations is written as follows:

$$
H=\int\left[\sum_{j=1}^{3}\left(\left|A_{j}\right|_{t}^{2}+\left|\hat{L}_{j} A_{j}\right|^{2}-\varepsilon\left|A_{j}\right|^{2}\right)-\frac{3}{2}\left(A_{1} A_{2} A_{3}+A_{1}^{*} A_{2}^{*} A_{3}^{*}\right)\right] d^{2} r .
$$

Thus, we obtained equations that describe the initial stages of the development of instability of the surface of a conducting liquid in a near-critical field, when the small-angle approximation is valid and the main nonlinear interaction is the interaction of three standing waves that form a hexagonal lattice. Note that analogous equations describe the instability of the charged surface of liquid helium [8, 17].

\section{CRITERION FOR EXPLOSIVE INSTABILITY}

As is known, hexagonal structures on a charged surface of various liquids are characterized by a hard regime of excitation [7, 8]. For the set of equations (14)-(16), this means the possibility of an unbounded growth of the amplitude $A_{j}$ in a finite time period. Indeed, in the simplest case, when the amplitudes are independent of the spatial variables, are real, and are equal to one another, i.e., $A_{1}=A_{2}=A_{3}=A(t)$, the time evolution of the quantity $A$ is described by an ordinary differential equation with a quadratic nonlinearity

$$
A_{t t}=\varepsilon A+3 A^{2} / 2
$$

Because of its influence, the amplitude grows asymptotically $\left(A \rightarrow 4\left(t-t_{c}\right)^{-2}\right)$, under corresponding initial conditions, i.e., the magnitude of $A$ becomes infinite at the moment $t_{c}$. However, what seems to be obvious for spatially uniform (coordinate-independent) solutions requires to be proved in the case of arbitrary amplitudes $A_{1}, A_{2}$, and $A_{3}$. In particular, of a significant interest is the situation where the initial perturbation of the surface is localized in a certain region.

Let us show, using the method of differential inequalities, that the nonlinear interaction of amplitude $A_{j}$ in terms of the model (14)-(16) results in an explosive growth of perturbations 
of the surface of a conducting liquid and find the sufficient conditions for hard excitation of the instability. To this end, we introduce the norm

$$
X_{j}(t)=\int\left|A_{j}\right|^{2} d^{2} r, \quad j=1,2,3
$$

and consider the time evolution of the following nonnegative quantity:

$$
X=\sum_{j=1}^{3} X_{j}
$$

By analogy with [12], we doubly differentiate $X$ with respect to $t$

$$
\begin{gathered}
X_{t t}=\sum_{j=1}^{3} \int\left(2\left|A_{j}\right|_{t}^{2}+A_{j t} A_{j}^{*}+A_{j t t}^{*} A_{j}\right) d^{2} r \\
=\int\left[2 \sum_{j=1}^{3}\left(\left|A_{j}\right|_{t}^{2}+\left|\hat{L}_{j} A_{j}\right|^{2}+\varepsilon\left|A_{j}\right|^{2}\right)+\frac{9}{2}\left(A_{1} A_{2} A_{3}+A_{1}^{*} A_{2}^{*} A_{3}^{*}\right)\right] d^{2} r,
\end{gathered}
$$

after substituting the corresponding right-hand sides of the amplitude equations (14)-(16) for the multiple derivatives $A_{j_{t t}}$ and $A_{j_{t t}}^{*}$. Then, eliminating the signambiguous cubic nonlinearity from the integrand using the expression for the Hamiltonian (17), we obtain the following relation:

$$
X_{t t}+3 H=-\varepsilon X+5 \sum_{j=1}^{3} \int\left[\left|A_{j}\right|_{t}^{2}+\left|\hat{L}_{j} A_{j}\right|^{2}\right] d^{2} r
$$

Now, our problem is to approximate the right-hand side of Eq. (18) using the magnitude of $X$ and thereby obtain an ordinary differential inequality. From the known CauchyBunyakowsky integral inequality for the functions $\left|A_{j}\right|$ and $\left|A_{j}\right|_{t}$,

$$
\left[\int\left|A_{j}\right|^{2} d^{2} r\right] \cdot\left[\int\left|A_{j}\right|_{t}^{2} d^{2} r\right] \geq\left[\int\left|A_{j}\right| \cdot\left|A_{j}\right|_{t} d^{2} r\right]^{2},
$$

it follows that $\int\left|A_{j}\right|_{t}^{2} d^{2} r \geq X_{j_{t}}^{2} /\left(4 X_{j}\right)$. Taking also into account the obvious relations $\int\left|\hat{L}_{j} A_{j}\right|^{2} d^{2} r \geq 0$ for $j=1,2,3$, we obtain from Eq. (18)

$$
X_{t t}+3 H \geq-\varepsilon X+\frac{5}{4} \sum_{j=1}^{3} \frac{X_{j_{t}}^{2}}{X_{j}}
$$

Then, note that, as a consequence of the algebraic Cauchy inequality, the following relation is valid:

$$
\left[\sum_{j=1}^{3} X_{j}\right] \cdot\left[\sum_{j=1}^{3} X_{j_{t}}{ }^{2} / X_{j}\right] \geq\left[\sum_{j=1}^{3} X_{j_{t}}\right]^{2}
$$


and, correspondingly, we have

$$
\sum_{j=1}^{3} X_{j_{t}}^{2} / X_{j} \geq X_{t}^{2} / X
$$

Substituting the latter inequality into (19), we obtain an ordinary differential inequality

$$
X_{t t}+3 H \geq-\varepsilon X+\frac{5}{4} \frac{X_{t}^{2}}{X}
$$

which will be the object of our consideration below. Note that analogous inequalities arise when deriving sufficient collapse criteria for various nonlinear partial differential equations [10, 11, 12, 13, 14].

The introduction of a new function $Y=X^{-1 / 4}$ permits us to rewrite the inequality (20) in the form of Newton's second law

$$
Y_{t t} \leq-\frac{\partial P(Y)}{\partial Y}, \quad P(Y)=-\frac{1}{8}\left(\varepsilon Y^{2}+H Y^{6}\right),
$$

where $Y$ plays the role of the coordinate of a "particle" and $P$ is its potential energy.

Let the velocity of the "particle" $Y_{t}$ be negative (in this case $X_{t}>0$ ). Then, multiplying (21) by $Y_{t}$, we obtain

$$
U_{t}(t) \geq 0, \quad U(t)=Y_{t}^{2} / 2+P(Y)
$$

i.e., the "particle" gains an energy $U$ upon motion. It is understandable that the sufficient criterion for $Y$ to become zero and, correspondingly, for $X$ to become infinity is the condition that the "particle" encounters no potential barrier even if $U_{t}=0$, which corresponds to the equality sign in (2). The explosive growth of amplitudes takes place under the following conditions:

(a) at $\varepsilon<0$ and $H>0$ if $Y\left(t_{0}\right)<|\varepsilon|^{\frac{1}{4}} /(3)^{\frac{1}{4}}$ and $12 U\left(t_{0}\right) \leq|\varepsilon|^{\frac{3}{2}} /(3 H)^{\frac{1}{2}}$;

(b) at $\varepsilon<0$ and $H>0$ if $12 U\left(t_{0}\right)>|\varepsilon|^{\frac{3}{2}} /(3 H)^{\frac{1}{2}}$;

(c) at $\varepsilon<0$ and $H \leq 0$;

(d) at $\varepsilon \geq 0$ if $U\left(t_{0}\right)>0$,

In (a)-(d), $t=t_{0}$ corresponds to the starting time moment. In this case, the moment $t_{c}$, at which the perturbation amplitudes become infinite is estimated as follows:

$$
t_{c} \leq t_{0}+\int_{0}^{Y\left(t_{0}\right)} \frac{d Y}{\sqrt{2 U\left(t_{0}\right)-2 P(Y)}} .
$$


Note that the condition $Y_{t}\left(t_{0}\right)<0$ in cases (a) and (c) is by no means necessary: after the reflection from a potential wall, the "particle" reaches the point $Y=0$. The above conditions (a)-(d) can be considered as sufficient criteria of the instability of the surface of a conducting liquid with respect to perturbations of a finite amplitude, which distinguishes it from the simplest criterion of linear instability $E>E_{c}$, which was derived based on the assumption that the perturbations are infinitely small. Note also that conditions (a)-(c) refer to the case of subcritical external fields $\left(E>E_{c}\right)$, when the flat surface of the conducting liquid is stable in the linear approximation, i.e., we deal with a hard excitation of an electrohydrodynamic instability.

Thus, if conditions (a)-(d) are fulfilled, Eqs. (14)-(16) describe an infinite growth of amplitudes $A_{j}$. In this case, the applicability of the model (14)-(16) to the description of the development of an electrohydrodynamic instability is restricted by the condition of the smallness of the amplitudes: in the order of magnitude, the absolute values of the amplitudes $\left|A_{j}\right|$ should not exceed the magnitude of the parameter of supercriticality $\varepsilon$. Otherwise, the model cannot be restricted to the consideration of only three-wave processes. As to the higher-order wave processes, there arises a question of whether they will lead to a stabilization of the instability or will favor an explosive growth of perturbations (the experimental data of [18] and the results of numerical calculations [3, 4] evidence in favor of the latter situation). The complexity of the estimation of their influence is related to the fact that the contribution of higherorder nonlinearities becomes comparable with the contribution of quadratic nonlinearities of the model (14)-(16), only if the amplitude of surface perturbations is close to the characteristic length of the wave. But in this case conditions of the applicability of our approach to the description of the near-critical dynamics of the charged surface of liquid metal based on the construction of amplitude equations become violated. Nevertheless, it is possible to reveal the influence of the higher-order nonlinearities by considering one-dimensional and square lattices of surface distributions, for which the three-wave interactions degenerate and the dominating interactions are the four-wave ones. 


\section{FOUR-WAVE INTERACTIONS}

Let us consider perturbations of the boundary of a conducting liquid with such symmetries for which the effect of three-wave processes is negligible. Thereby, we in pure form separate the four-wave interactions that determine the character of the electrohydrodynamic instability at its advanced stages.

First of all, we consider the near-critical behavior of the charged surface of a liquid metal in the assumption of a quasi-one-dimensional character of the arising wave. Let the wave vector be parallel to the abscissa axis. We pass to envelopes by using substitutions

$$
\begin{aligned}
& \eta(x, y, t)=A(x, y, t) e^{i k_{0} x}+A_{0}(x, y, t) e^{2 i k_{0} x}+\text { c.c. } \\
& \psi(x, y, t)=B(x, y, t) e^{i k_{0} x}+B_{0}(x, y, t) e^{2 i k_{0} x}+\text { c.c. }
\end{aligned}
$$

in which the $k_{0} \leftrightarrow 2 k_{0}$ interaction is taken into account. Here, $A, B, A_{0}$ and $B_{0}$ are slowly varying functions of the spatial variables $x$ and $y$. Substituting these relations into the Hamiltonian (12), we find (to an accuracy of terms of the fourth order of smallness)

$$
\begin{gathered}
H=H^{(2)}+H^{(3)}+H^{(4)} \\
H^{(2)}=\int\left[k_{0}|B|^{2}-2 g \varepsilon|A|^{2}+\frac{g}{k_{0}^{2}}\left|\frac{\partial A}{\partial x}-\frac{i}{2 k_{0}} \frac{\partial^{2} A}{\partial y^{2}}\right|^{2}+g\left|A_{0}\right|^{2}+2 k_{0}\left|B_{0}\right|^{2}\right] d^{2} r \\
H^{(3)}=-2 g k_{0} \int\left[A^{2} A_{0}^{*}+A^{* 2} A_{0}\right] d^{2} r \\
H^{(4)}=\frac{5}{4} g k_{0}^{2} \int|A|^{4} d^{2} r .
\end{gathered}
$$

The amplitude equations for the perturbations of the free surface are written in the Hamiltonian form as

$$
\frac{\partial A}{\partial t}=\frac{\delta H}{\delta B^{*}}, \quad \frac{\partial B}{\partial t}=-\frac{\delta H}{\delta A^{*}}, \quad \frac{\partial A_{0}}{\partial t}=\frac{\delta H}{\delta B_{0}{ }^{*}}, \quad \frac{\partial B_{0}}{\partial t}=-\frac{\delta H}{\delta A_{0}{ }^{*}} .
$$

By varying the function $H$, and then eliminating the quantities $B$ and $B_{0}$, we obtain equations of the form

$$
\begin{gathered}
A_{t t}=2 g k_{0} \varepsilon A+\frac{g}{k_{0}}\left(\frac{\partial}{\partial x}-\frac{i}{2 k_{0}} \frac{\partial^{2}}{\partial y^{2}}\right)^{2} A+4 g k_{0}^{2} A^{*} A_{0}-\frac{5}{2} g k_{0}^{3} A^{2} A^{*} \\
A_{0 t t}=-2 g k_{0} A_{0}+4 g k_{0}^{2} A^{2} .
\end{gathered}
$$


Since the characteristic times of changes in the amplitudes at small supercriticalities are small $\left(\omega^{2} \sim \varepsilon\right)$, we neglect the derivatives with respect to time in Eq. (23). Then, the quantity $A_{0}$ can be expressed through the amplitude $A$ that plays the role of an order parameter

$$
A_{0} \approx 2 k_{0} A^{2}
$$

Using this relation, we eliminate $A_{0}$ from Eq. (22) and pass to dimensionless quantities using scalings (13) to obtain for the complex amplitude $A$ :

$$
A_{t t}=\varepsilon A+\hat{L}_{1}^{2} A+s A|A|^{2}, \quad s=11 / 4
$$

to which the following expression for the Hamiltonian corresponds:

$$
H=\int\left[\left|A_{t}\right|^{2}+\left|\hat{L}_{1} A\right|^{2}-\varepsilon|A|^{2}-s|A|^{4} / 2\right] d^{2} r .
$$

Note that, when neglecting the dependence of the amplitude $A$ on $y$, Eq. (24) becomes a nonlinear Klein-Gordon equation, i.e., corresponds to the so-called $|\psi|^{4}$ model. In this form, it can be obtained from the equation for one-dimensional perturbations of the charged surface of liquid helium [8] in the limit of the complete shielding of the field under the surface. Note also that if we neglect transverse modulations, then Eq. (24) coincides with that obtained in the Kelvin-Helmholtz theory of instability for the case of a small ratio of the densities of the top and bottom liquids [12]. This is due to the identity of the mathematical description of the planar potential flow of an incompressible liquid and a two-dimensional distribution of an electric field in the absence of spatial electric charges. The allowance for higher-order terms in the expansions in surface perturbations violates this analogy.

Since the term $\left|A_{t}\right|^{2}$ that is responsible for the kinetic energy and the term $|A|^{4}$ that is responsible for the fourwave processes enter into the integrand of the Hamiltonian (25) with the opposite signs, Eq. (24) admits infinite solutions. This means that the cubic nonlinearity in Eq. (24) does not stabilize the linear instability but, on the contrary, enhances it, leading, under certain conditions, to an explosive growth of the amplitude $A$ of perturbations of the conducting-liquid boundary.

Another possible case when the three-wave processes are degenerate is the interaction of two standing waves whose wave vectors $\mathbf{k}_{1}$ and $\mathbf{k}_{2}$ are turned with respect to one another by an angle $\pi / 2$ (the vector's coordinates are $\mathbf{k}_{1}=\left\{k_{0}, 0\right\}$ and $\mathbf{k}_{2}=\left\{0, k_{0}\right\}$ ). Let us represent 
the perturbation of the surface $\eta$ in the form

$$
\begin{gathered}
\eta(\mathbf{r}, t)=\sum_{j=1}^{2}\left[A_{j} e^{i \mathbf{k}_{j} \mathbf{r}}+2 k_{0} A_{j}^{2} e^{2 i \mathbf{k}_{j} \mathbf{r}}\right] \\
+(12 \sqrt{2}+16) k_{0}\left[A_{1} A_{2} e^{i\left(\mathbf{k}_{1}+\mathbf{k}_{2}\right) \mathbf{r}}+A_{1} A_{2}^{*} e^{i\left(\mathbf{k}_{1}-\mathbf{k}_{2}\right) \mathbf{r}}\right]+\text { c.c. }
\end{gathered}
$$

and the perturbation of the velocity potential at the liquid boundary $\psi$ in the form

$$
\begin{gathered}
\psi(\mathbf{r}, t)=\sum_{j=1}^{2}\left[k_{0}^{-1} A_{j t} e^{i \mathbf{k}_{j} \mathbf{r}}+2 A_{j} A_{j t} e^{2 i \mathbf{k}_{j} \mathbf{r}}\right] \\
+(6+4 \sqrt{2})\left[\left(A_{1} A_{2}\right)_{t} e^{i\left(\mathbf{k}_{1}+\mathbf{k}_{2}\right) \mathbf{r}}+\left(A_{1} A_{2}{ }^{*}\right)_{t} e^{i\left(\mathbf{k}_{1}-\mathbf{k}_{2}\right) \mathbf{r}}\right]+\text { c.c. },
\end{gathered}
$$

where we took into account the nonlinear interactions of the fundamental spatial harmonic $k_{0}$ with mixed harmonics $2 k_{0}$ and $\sqrt{2} k_{0}$. This representation for the functions $\eta$ and $\psi$ corresponds to the symmetry of a square lattice.

Proceeding by analogy to the above-considered quasi-one-dimensional case, we obtain, after passing to dimensionless quantities, the following dynamic equations:

$$
\begin{aligned}
& A_{1 t t}=\varepsilon A_{1}+\hat{L}_{1}^{2} A_{1}+s A_{1}\left|A_{1}\right|^{2}+\sigma A_{1}\left|A_{2}\right|^{2}, \\
& A_{2 t t}=\varepsilon A_{2}+\hat{L}_{2}^{2} A_{2}+s A_{2}\left|A_{2}\right|^{2}+\sigma A_{2}\left|A_{1}\right|^{2},
\end{aligned}
$$

where $\sigma=32 \sqrt{2}+65 / 2$, and the following designations are introduced:

$$
\hat{L}_{1}=\frac{\partial}{\partial x}-\frac{i}{\sqrt{2}} \frac{\partial^{2}}{\partial y^{2}} \quad \text { and } \quad \hat{L}_{2}=\frac{\partial}{\partial y}-\frac{i}{\sqrt{2}} \frac{\partial^{2}}{\partial x^{2}} .
$$

The integral of motion for these equations, corresponding to the conservation of the total energy of a conservative system, is given by the expression

$$
H=\sum_{j=1}^{2} \int\left(\left|A_{j}\right|_{t}^{2}+\left|\hat{L}_{j} A_{j}\right|^{2}-\varepsilon\left|A_{j}\right|^{2}-s\left|A_{j}\right|^{4} / 2\right) d^{2} r-\sigma \int\left|A_{1}\right|^{2}\left|A_{2}\right|^{2} d^{2} r
$$

The first term on the right-hand side of this functional coincides in its structure with the Hamiltonian (25) for the quasi-one-dimensional wave. The last term is responsible for the nonlinear interaction of a pair of the waves studied. Note that the coefficient $\sigma$ before this term exceeds the coefficient $s$ by more than an order of magnitude. This means that the contribution of the interaction $\mathbf{k}_{1} \leftrightarrow \mathbf{k}_{2}$ is determining and, consequently, the square structure of the surface perturbations is much more favorable than the one-dimensional one. 
In any case, for both the square and one-dimensional lattice (the latter can be considered as a partial case of the square lattice, corresponding to the condition $A_{2}=0$ ), the four-wave interactions will favor the development of an instability rather than suppress it. Conditions for an explosive growth of the amplitudes $A_{1}$ and $A_{2}$ can be obtained by considering the evolution of the norm

$$
X=\int\left(\left|A_{1}\right|^{2}+\left|A_{2}\right|^{2}\right) d^{2} r .
$$

Acting by analogy with Section 5, we obtain the majorizing inequality

$$
X_{t t}+4 H \geq-2 \varepsilon X+\frac{3}{2} \frac{X_{t}^{2}}{X},
$$

which coincides with that considered in [12]. The introduction of the variable $Y=X^{-1 / 2}$ reduces the problem to the analysis of the motion of a "particle" with a coordinate $Y$ in a potential well $P(Y)$

$$
Y_{t t} \leq-\frac{\partial P(Y)}{\partial Y}, \quad P(Y)=-\frac{1}{2}\left(\varepsilon Y^{2}+H Y^{4}\right) .
$$

Analyzing this inequality for the case where the velocity of the "particle" at the initial time moment $t=t_{0}$ is directed toward the origin (i.e., $Y_{t}\left(t_{0}\right)<0$ ), it can easily be revealed that the quantity $Y$ vanishes, first, at $\varepsilon>0$, if $U\left(t_{0}\right)>0$, second, at $\varepsilon<0$ and $H<0$, and third, at $\varepsilon<0$ and $H>0$, if $U\left(t_{0}\right)>\varepsilon^{2} /(8 H)$ or $Y^{2}\left(t_{0}\right)<|\varepsilon| /(2)$. Here, $U(Y)$, just as in Section 5 , denotes the total mechanical energy of the "particle." Under the above conditions, the norm $X$ become infinite in a finite time, which just corresponds to an explosive growth of the amplitudes in the result of four-wave interactions.

All this suggests that the higher-order nonlinearities will not suppress the explosive growth of amplitudes in the model (14)-(16). But in this case the above integral criteria (a)-(d) may be considered as sufficient criteria of the explosive growth of perturbations of the surface of a liquid metal in an external electric field.

\section{CONCLUSION}

The main result of this work is the construction of sufficient integral criteria of instability for the free surface of an ideally conducting liquid in a near-critical external electric field. These criteria represent a generalization of the known condition of linear instability $\left(E>E_{c}\right)$ to the case where the amplitudes of perturbations of the field of velocities and of the shape 
of the surface are finite. The criteria found are dynamic in the sense that they take into account the effect of the velocity distribution in the medium at the initial time moment; the role of the stored kinetic energy can be decisive in the case of the hard mechanism of instability.

An analysis of three-wave and four-wave nonlinear interactions (this corresponds to the allowance for quadratic and cubic nonlinearities in the amplitude equations) showed that the development of the electrohydrodynamic instability has an explosive character, i.e., leads to the appearance of singularities in the solutions in a finite time. This conclusion qualitatively agrees with the results of numerical simulation of the development of the instability of the boundary of liquid metal: it was shown in [4] that the curvature of the surface increases according to a power law characteristic of the explosive instability and causes the formation of specific features of a cusplike type.

Note in conclusion that the criteria of hard instability analogous to the criteria (a)-(d) can also be obtained for insulating liquids with induced surface charges [9], for insulating liquids with free surface charges (liquid helium and liquid hydrogen in an electric field refer to this category) [9, 19], and for ferromagnetic liquids in a vertical magnetic field.

This work was supported in part by the Russian Foundation for Basic Research (project no. 00-02- 17428) and by the INTAS (project no. 99-1068). We are grateful to N.B. Volkov and A.M. Iskol'dskii for interest in our work; N.M. Zubarev is also grateful to E.A. Kuznetsov for stimulating discussions.

[1] L. Tonks, Phys. Rev., 48, 562 (1935).

[2] Ya.I. Frenkel, Zh. Eksp. Teor. Fiz., 6(4), 350 (1936).

[3] A.L. Pregenzer and B.M. Marder, J. Appl. Phys., 60, 3821 (1986).

[4] V.G. Suvorov and E.A. Litvinov, J. Phys. D: Appl. Phys., 33, 1245 (2000).

[5] N.M. Zubarev, Zh. Eksp. Teor. Fiz., 114, 2043 (1998) [JETP, 87, 1110 (1998)].

[6] L.D. Landau and E.M. Lifshitz, Course of Theoretical Physics, Vol. 8: Electrodynamics of Continuous Media (Nauka, Moscow, 1982; Pergamon, New York, 1984).

[7] E.A. Kuznetsov and M.D. Spektor, Zh. Eksp. Teor. Fiz., 71(1), 262 (1976) [Sov. Phys. JETP 44, 136 (1976)]. 
[8] L.P. Gor'kov and D.M. Chernikova, Dokl. Akad. Nauk SSSR, 228, 829 (1976) [Sov. Phys. Dokl., 21, 328 (1976)].

[9] N.M. Zubarev and O.V. Zubareva, Phys. Lett. A, 272, 119 (2000).

[10] E.A. Kuznetsov, J.J. Rasmussen, K. Rypdal, and S.K. Turitsyn, Physica D, 87, 273 (1995).

[11] P.M. Lushnikov, Pis'ma Zh. Eksp. Teor. Fiz., 62, 447 (1995) [JETP Lett. 62, 461 (1995)].

[12] E.A. Kuznetsov and P.M. Lushnikov, Zh. Eksp. Teor. Fiz., 108, 614 (1995) [JETP, 81, 332 (1995)].

[13] E.M. Maslov and A.G. Shagalov, Phys. Lett. A., V.239, 46 (1998).

[14] S.K. Turitsyn, Phys. Rev. E., 47, R796 (1993).

[15] V.E. Zakharov, Prikl. Mekh. Tekh. Fiz., (2), 86, (1968).

[16] V.E. Zakharov and E.A. Kuznetsov, Usp. Fiz. Nauk, 167, 1137 (1997) [Phys. Usp., 40, 1087 (1997)].

[17] D.M. Chernikova, Fiz. Nizk. Temp., 6, 1513 (1980) [Sov. J. Low Temp. Phys., 6, 737 (1980)].

[18] M.D. Gabovich and V.Ya. Poritskii, Pis'ma Zh. Eksp. Teor. Fiz., 33, 320 (1981) [JETP Lett. 33, 304 (1981)].

[19] N.M. Zubarev and O.V. Zubareva, Pis'ma Zh. Tekh. Fiz., 26(9), 65 (2000) [Tech. Phys. Lett., 25, 872 (1999)]. 